\title{
THE ORBIT OF THE SPECTROSCOPIC BINARY $\eta$ PEGASI
}

This orbit of $\eta$ Pegasi is based upon the twentynine observations given above by Director CAMPBELL. Of these, several groups have been formed into normal positions by taking the mean of their dates and of their velocities. These groups are Nos. 5, 6, 7, 8; $9,10,11 ; 14,15 ; 16,17$; and 20,21 . To these will be given the numbers $\mathrm{N}_{1}, \mathrm{~N}_{2}$, etc. Their data are as follows:

\begin{tabular}{|c|c|c|c|c|c|c|c|}
\hline No. & $\begin{array}{c}\text { Date. } \\
\text { Greenwich M.T }\end{array}$ & & & & & $\begin{array}{l}\text { VELOCITY. } \\
\text { Kilometers. }\end{array}$ & WT \\
\hline $\mathrm{N}_{1}$ & 1898 September & 4.8 & . & . & 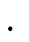 &.+16.09 & 4 \\
\hline $\mathrm{N}_{2}$ & October & 23.4 & . & . & $0^{\circ}$ &.+11.11 & 3 \\
\hline $\mathrm{N}_{3}$ & 1899 May & 5.5 & . & . & ${ }^{\circ}$ & . -6.19 & \\
\hline $\mathrm{N}_{4}$ & June & 24.0 & . & . & $\cdot$ & . -8.16 & \\
\hline $\mathrm{N}_{5}$ & December & 22.7 & . & . & . & . -2.65 & 2 \\
\hline
\end{tabular}

All of the other observations have been given weight unity, except No. 13, to which, for reasons given by Director CAMPBELL, weight one-half has been assigned.

From the plot of these observations the first approximation to the period was taken to be 815 days. By adjusting the upper and the lower areas to equality by means of a planimeter, the velocity of the center of mass of the system was found to be $+4.15^{\mathrm{km}}$.

Using the formulae and notation of LEHMANNFilhés, the following preliminary constants and elements have been obtained:

\section{Prelininary Elements.}

$$
\begin{array}{rl}
\mathrm{A}=16.85^{\mathrm{km}} & \mathrm{B}=12.05^{\mathrm{km}} \\
\mathrm{z}_{1}=+0.429 & \quad \mathrm{z}_{2}=-0.482 \\
\mathrm{~K} & =14.45 \quad \\
\mathrm{e} & =0.176 \\
\omega & =19.056 \\
\mu & =0.007709 \mathrm{rad} . \\
& =0.4417 \\
\mathrm{~T} & =1898 \text { Aug. } 3.0 \\
\mathrm{~V}_{0} & =+4.15^{\mathrm{km}} \\
\mathrm{U} & =815.0 \text { days } \\
\mathrm{a} \sin \mathrm{i} & =159,420,000 \\
{[\mathrm{pvv}]_{\mathrm{n}}} & =19.55
\end{array}
$$

The residuals resulting from these elements are given in the table at the end. From these elements and the residuals, the following twenty-one weighted, homogeneous observation equations were set up:

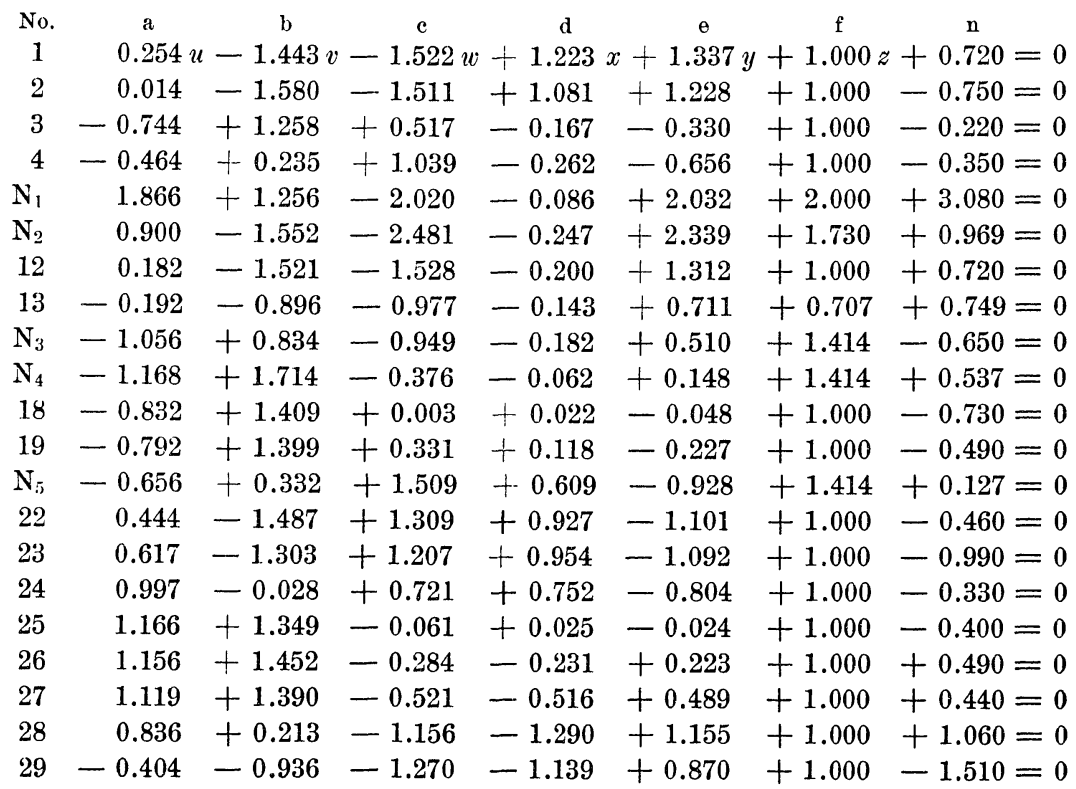

where $u=\delta \mathrm{K} ; v=10 \delta \mathrm{e} ; w=10 \delta \omega ; x=10,000 \delta \mu$; $y=\frac{1}{10} \delta \mathrm{T} ;$ and $z=\delta \mathrm{V}_{\mathrm{o}}$.

From these equations the following normal equa- tions result (the coefficients are given by their logarithms) : $\begin{array}{ll}1 & 1.19882 u+0.322426_{\mathrm{n}} v+0.817499_{\mathrm{n}} w+9.397940 x+0.809492 y+0.665675 z+0.955303=0 \\ 2 & 0.322426_{\mathrm{n}}+1.501004+0.756408+0.760875_{\mathrm{n}}+0.670060_{\mathrm{n}}+0.565021+0.544068=0 \\ 3 & 0.817499_{\mathrm{n}}+0.756408+1.475599+0.673021+1.405858_{\mathrm{n}}+1.060244_{\mathrm{n}}+1.084219_{\mathrm{n}}=0 \\ 4 & 9.397940+0.760875_{\mathrm{n}}+0.673021+0.950754+0.609914_{\mathrm{n}}+0.046495+0.320977_{\mathrm{n}}=0 \\ 5 & 0.809492+0.670060_{\mathrm{n}}+1.405858_{\mathrm{n}}+0.609914_{\mathrm{n}}+1.348110+1.023828+1.087320=0 \\ 6 & 0.665675+0.565021+1.060244_{\mathrm{n}}+0.046495+1.023828+1.454692+0.747179=0\end{array}$

$-27-$ 
The solution of these gives:

$$
\begin{array}{ll}
\delta \mathrm{K}=-0.29^{\mathrm{km}} & \pm 0.12^{\mathrm{km}} \\
\delta \mathrm{e}=-0.0264 & \pm 0.0091 \\
\delta \omega=-11.143 & \pm 2.822 \\
\delta \mu=-0.000023 \mathrm{rad} . & \pm 0.000018 \mathrm{rad} \\
\multicolumn{1}{c}{=-0.00132} & \pm 0.00101 \\
\delta \mathrm{T}=-28.6 \text { days } & \pm 5.9 \text { days } \\
\delta \mathrm{V}_{\mathrm{o}}=+0.17^{\mathrm{km}} & \pm 0.10^{\mathrm{km}} \\
\delta \mathrm{U}=+2.4+\text { days } & \pm 1.9 \text { days }
\end{array}
$$

From these corrections the following elements result:

$$
\begin{aligned}
& \text { SECOND Elements. } \\
& \mathrm{K}=14.16^{\mathrm{km}} \\
& \mathrm{e}=0.1496 \\
& \omega=7^{\circ} .913 \\
& \mu=0.007686 \mathrm{rad} . \\
& =0.44038 \\
& \mathrm{~T}=1898 \text { July } 5.4 \\
& \mathrm{~V}_{\mathrm{o}}=+4.32^{\mathrm{km}} \\
& \mathrm{U}=817.4+\text { days }
\end{aligned}
$$

$[\mathrm{pvv}]_{\mathrm{n}}=6.05$ from the observation equations. The probable errors given above were obtained from this $[\mathrm{pvv}]_{\mathrm{n}}$.

$[p v v]_{\mathrm{n}}=6.55$ from the ephemeris residuals.

$[\mathrm{pvv}]=11.33$.
Note.- $[\mathrm{pvv}]_{\mathrm{n}}$ means the $[\mathrm{p} v \mathrm{v}]$ resulting from using the residuals from the normal places instead of from the individual observations. When the latter are used no subscript will

\begin{tabular}{|c|c|c|c|c|c|c|c|}
\hline No. & a & b & c & $\mathrm{d}$ & e & $f$ & $n$ \\
\hline 1 & 0 . & $1.472 v$ & $-1.442 w$ & +1 & 1 & + & $30=0$ \\
\hline 2 & -0.007 & -1.455 & -1.428 & +0.935 & +1.106 & +1.000 & $-0.880=0$ \\
\hline 3 & -0.766 & +1.113 & +0.537 & -0.155 & -0.330 & +1.000 & $-0.160=0$ \\
\hline 4 & -0.470 & -0.092 & +1.084 & -0.256 & -0.705 & +1.000 & $-0.120=0$ \\
\hline $\mathrm{N}_{1}$ & 1.730 & +0.266 & -2.034 & -0.158 & +1.972 & +2.000 & $+0.940=0$ \\
\hline $\mathrm{N}_{2}$ & 0.826 & -1.931 & -2.366 & -0.321 & +2.101 & +1.732 & $-0.052=0$ \\
\hline 12 & 0.167 & -1.492 & -1.445 & -0.224 & +1.179 & +1.000 & $+0.630=0$ \\
\hline 13 & -0.182 & -0.713 & -0.936 & -0.176 & +0.669 & +0.707 & $+1.075=0$ \\
\hline $\mathrm{N}_{3}$ & -1.046 & +0.966 & -0.960 & -0.223 & +0.563 & +1.414 & $+0.042=0$ \\
\hline $\mathrm{N}_{4}$ & -1.181 & +1.769 & -0.402 & -0.097 & +0.211 & +1.414 & $+0.919=0$ \\
\hline 18 & -0.852 & +1.406 & -0.015 & +0.004 & -0.008 & +1.000 & $-0.600=0$ \\
\hline 19 & -0.820 & +1.327 & +0.323 & +0.113 & -0.203 & +1.000 & $-0.490=0$ \\
\hline $\mathrm{N}_{5}$ & -0.682 & -0.068 & +1.513 & +0.683 & -0.980 & +1.414 & $+0.198=0$ \\
\hline 22 & 0.490 & -1.301 & +1.302 & +0.992 & -1.125 & +1.000 & $+0.370=0$ \\
\hline 23 & 0.677 & -0.910 & +1.172 & +0.982 & -1.075 & +1.000 & $-0.150=0$ \\
\hline 24 & 1.034 & +0.575 & +0.627 & +0.647 & -0.657 & +1.000 & $+0.070=0$ \\
\hline 25 & 1.145 & +1.417 & -0.147 & -0.131 & +0.124 & +1.000 & $-0.870=0$ \\
\hline 26 & 1.121 & +1.336 & -0.354 & -0.367 & +0.341 & +1.000 & $-0.170=0$ \\
\hline 27 & 1.073 & +1.109 & -0.567 & -0.614 & +0.561 & +1.000 & $-0.360=0$ \\
\hline 28 & 0.788 & -0.178 & -1.117 & -1.235 & +1.067 & +1.000 & $+0.310=0$ \\
\hline 29 & -0.371 & -0.802 & -1.239 & -1.149 & +0.851 & +1.000 & $-0.760=0$ \\
\hline
\end{tabular}
appear with the symbol [pvv].

The resulting probable error of a single observation is:

$$
\begin{aligned}
& r= \pm 0.43^{\mathrm{km}} \text { from the first }[\mathrm{pvv}]_{\mathrm{n}} . \\
& r= \pm 0.474^{\mathrm{km}} \text { from }[\mathrm{pvv}] .
\end{aligned}
$$

The velocities and the corresponding residuals computed from these elements are shown in the table at the end. This table gives also the differences between the residuals as found from the elements and from the observation equations. This column is headed $\Delta_{1}$. From these differences it is seen that the terms involving the second powers of the increments in the differential equations of condition are not negligible. This discrepancy necessitated another solution, starting from the second elements.

From these elements the following weighted, homogeneous observation equations result: - where, as before, $u=\delta \mathrm{K} ; v=10 \delta \mathrm{e} ; w=10 \delta \omega$; $x=10,000 \delta \mu ; y=\frac{1}{10} \delta \mathrm{T} ;$ and $z=\delta \mathrm{V}_{\mathrm{o}}$.
From these equations the following normal equations were computed (coefficients logarithmic) :-

$\begin{array}{ll}1 & 1.181443 u+0.590730_{\mathrm{n}} v+0.783868_{\mathrm{n}} w+9.398808_{\mathrm{n}} x+0.759441 y+0.609594 z+9.680789=0 \\ 2 & 0.590730_{\mathrm{n}}+1.453624+0.886378+0.635685_{\mathrm{n}}+0.798547_{\mathrm{n}}+0.015360+0.308137_{\mathrm{n}}=0 \\ 3 & 0.783868_{\mathrm{n}}+0.886378+1.454387+0.765929+1.372258_{\mathrm{n}}+1.053981_{\mathrm{n}}+0.283075 \mathrm{n}=0 \\ 4 & 9.398808_{\mathrm{n}}+0.635685_{\mathrm{n}}+0.765929+0.932068+0.704751_{\mathrm{n}}+9.033424+9.155336=0 \\ 5 & 0.759441+0.798547_{\mathrm{n}}+1.372258_{\mathrm{n}}+0.704751_{\mathrm{n}}+1.299202+1.003590+0.252125=0 \\ 6 & 0.609594+0.015360+1.053981_{\mathrm{n}}+9.033424+1.003590+1.454799+0.093071=0\end{array}$


The solution of these gives:-

$$
\begin{aligned}
& \delta \mathrm{K}=+0.04^{\mathrm{km}} \quad \pm 0.12^{\mathrm{km}} \quad \pm 0.13^{\mathrm{km}} \\
& \delta_{\mathrm{e}}=+0.0052 \quad \pm 0.0098 \quad \pm 0.0106 \\
& \delta \omega=-2.308 \quad \pm 3.430 \quad \pm 3.708 \\
& \delta \mu=-0.000005 \mathrm{rad} . \quad \pm 0.000019 \mathrm{rad} . \quad \pm 0.000020 \mathrm{rad} \\
& =-0.00029 \quad \pm 0.00108 \quad \pm 0.00117 \\
& \delta \mathrm{T}=-5.7 \text { days } \quad \pm 7.5 \text { days } \quad \pm 8.1 \text { days } \\
& \delta \mathrm{V}_{\mathrm{o}}=-0.01^{\mathrm{km}} \quad \pm 0.10^{\mathrm{km}} \quad \pm 0.10^{5} \mathrm{~km} \\
& \delta \mathrm{U}=+0.5 \text { days } \quad \pm 2.0 \text { days } \quad \pm 2.2 \text { days } \\
& {[\mathrm{pvv}]_{\mathrm{n}}=6.21} \\
& {[\mathrm{pvv}]=11.13} \\
& r= \pm 0.43 \text { from }[\mathrm{pvv}]_{\mathrm{n}} \\
& r= \pm 0.469 \text { from [pvv] }
\end{aligned}
$$

The first set of probable errors given after the corrections to the elements has been derived from the first $r$; the second set from the second $r$. The latter, depending upon the residuals from the individual observations, is the more reliable.

Applying these corrections to the second elements given above, the following final elements result:-

The curve in the accompanying figure is that of the final elements. The observations are represented by the small circles. The intervals shown on the $X$ axis are 50 days. The zero is at the place representing the time of periastron $(T)$. One division on the
Final Elements.

$$
\begin{aligned}
\mathrm{K} & =14.20^{\mathrm{km}} & & \pm 0.13^{\mathrm{km}} \\
\theta & =0.1548 & & \pm 0.0106 \\
\omega & =5.605 & & \pm 3.708 \\
\mu & =0.007681 \mathrm{rad} . & & \pm 0.000020 \mathrm{rad} . \\
& =0.44009 & & \pm 0.00117 \\
\mathrm{~T} & =1898 \text { June } 29.7 & & \pm 8.1 \text { days } \\
& =1900 \text { Sept. } 25.7 & & \\
\mathrm{~V}_{0} & =+4.311^{\mathrm{km}} & & \pm 0.10^{\mathrm{km}} \\
\mathrm{U} & =818.0 \text { days } & & \pm 2.2 \text { days } \\
\text { a } \sin \mathrm{i} & =157.800,000 & &
\end{aligned}
$$

The velocities and the corresponding residuals computed from these elements are shown in the table at the end. The differences between the residuals as found from these elements and from the observation equations are placed in the column $\Delta_{2}$. They show this final solution to be satisfactory.

$Y$ axis represents a velocity of one kilometer per second. The dotted line indicates the velocity of the center of mass of the system. The maximum velocity is $+20.70 \mathrm{~km}$. per second and the minimum is $-7.70 \mathrm{~km}$. per second.

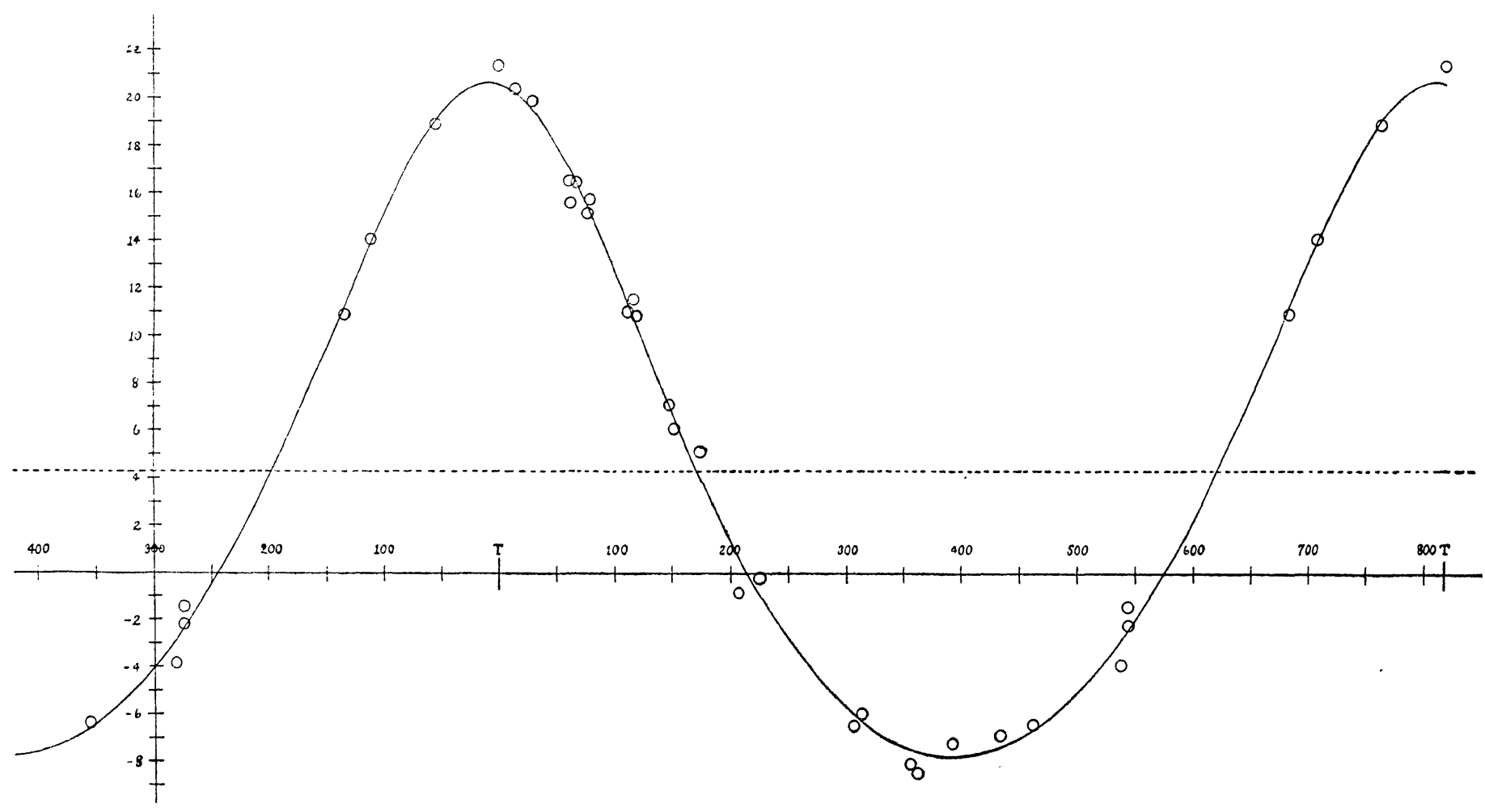


TABLE.

\begin{tabular}{|c|c|c|c|c|c|c|c|c|c|c|}
\hline \multirow{2}{*}{ No. } & \multirow{2}{*}{$\mathrm{V}$} & \multirow{2}{*}{ Wt. } & \multicolumn{2}{|c|}{ ELEMENTS-I. } & \multicolumn{2}{|c|}{ ELEMENTS-II. } & \multirow{2}{*}{$\Delta_{1}$} & \multicolumn{2}{|c|}{ ELEMENTS-III. } & \multirow{2}{*}{$\Delta_{2}$} \\
\hline & & & $\mathrm{v}$ & $\mathrm{O}-\mathrm{C}$ & $\mathrm{V}$ & $0-\mathrm{C}$ & & $\mathrm{v}$ & $\mathrm{O}-\mathrm{C}$ & \\
\hline & $\frac{\mathrm{km} .}{\mathrm{sec} .}$ & & $\frac{\mathrm{km}}{\mathrm{sec} .}$ & $\frac{\mathrm{km}}{\mathrm{sec} .}$ & $\frac{\mathrm{km}}{\text { sec. }}$ & $\frac{\mathrm{km}}{\text { sec. }}$ & & $\frac{\mathrm{km}}{\mathrm{sec}}$. & $\frac{\mathrm{km}}{\mathrm{sec} .}$ & \\
\hline 1 & +7.10 & 1 & +7.82 & -0.72 & $+\quad 7.33$ & -0.23 & 0.18 & $+\quad 7.10$ & \pm 0.00 & 0.00 \\
\hline 2 & +5.10 & 1 & +4.35 & +0.75 & +4.22 & +0.88 & 0.11 & +4.04 & +1.06 & 0.01 \\
\hline 3 & -6.37 & 1 & -6.59 & +0.22 & -6.53 & +0.16 & 0.02 & -6.57 & +0.20 & 0.04 \\
\hline 4 & -2.21 & 1 & -2.56 & +0.35 & -2.33 & +0.12 & 0.07 & $-\quad 2.39$ & +0.18 & 0.01 \\
\hline 5 & +16.54 & 1 & .................... & ..................... & +17.14 & -0.60 & $\ldots \ldots \ldots$ & +17.03 & -0.49 & $\cdots \cdot \cdot$ \\
\hline 6 & +15.62 & 1 & ................... & ……......... & +17.04 & -1.42 & ........ & +16.93 & -1.31 & the \\
\hline 7 & +16.46 & 1 & & (2) & +16.57 & -0.11 & & +16.45 & +0.01 & \\
\hline $\mathrm{N}_{1}$ & +16.09 & 4 & +17.63 & -1.54 & +16.56 & -0.47 & 0.12 & & & 0.04 \\
\hline 8 & +15.74 & 1 & & .................. & +15.44 & +0.30 & & +15.19 & +0.55 & \\
\hline 9 & +10.99 & 1 & & & +11.65 & -0.66 & & +11.47 & -0.48 & \\
\hline $\mathrm{N}_{2}$ & +11.11 & 3 & +11.67 & -0.56 & +11.08 & +0.03 & 0.21 & & & 0.01 \\
\hline 10 & +11.51 & 1 & & . ……… & +10.91 & +0.60 & & +10.73 & +0.78 & \\
\hline 11 & +10.83 & 1 & & & +10.67 & +0.16 & & +10.48 & +0.35 & \\
\hline 12 & +6.06 & 1 & +6.78 & -0.72 & $\begin{array}{r}+6.69 \\
+\quad 6 .\end{array}$ & -0.63 & 0.13 & $\begin{array}{r}+\quad 6.53 \\
+\end{array}$ & -0.47 & 0.00 \\
\hline 13 & -0.84 & $\frac{1}{2}$ & +0.22 & -1.06 & +0.68 & -1.52 & 0.01 & $+\quad 0.62$ & -1.46 & 0.00 \\
\hline 14 & -6.44 & 1 & & & -6.02 & -0.42 & & -5.97 & -0.47 & \\
\hline $\mathrm{N}_{3}$ & -6.19 & 2 & -6.65 & +0.46 & -6.16 & -0.03 & 0.05 & & & 0.00 \\
\hline 15 & -5.94 & 1 & 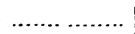 & ………........ & -6.30 & +0.36 & & -6.25 & +0.31 & \\
\hline 16 & -8.02 & 1 & & & -7.46 & -0.56 & & -7.41 & -0.61 & \\
\hline $\mathrm{N}_{4}$ & -8.16 & 2 & -7.78 & -0.38 & -7.51 & -0.65 & 0.05 & & & 0.00 \\
\hline 17 & -8.31 & 1 & & & -7.55 & -0.76 & & $-" 7.50$ & -0.81 & \\
\hline 18 & -7.14 & 1 & -7.87 & +0.73 & -7.74 & +0.60 & 0.04 & -7.70 & +0.56 & 0.00 \\
\hline 19 & -6.81 & 1 & -7.30 & +0.49 & -7.30 & +0.49 & 0.01 & -7.29 & +0.48 & 0.00 \\
\hline 20 & -3.86 & 1 & & & -2.71 & -1.15 & & -2.80 & -1.06 & \\
\hline $\mathrm{N}_{5}$ & -2.65 & 2 & -2.56 & -0.09 & -2.51 & -0.14 & 0.10 & & & 0.00 \\
\hline 21 & -1.44 & 1 & & & -2.30 & +0.86 & & $-\quad 2.39$ & +0.95 & \\
\hline 22 & +10.89 & 1 & +10.43 & +0.46 & +11.26 & -0.37 & 0.01 & +11.27 & -0.38 & 0.00 \\
\hline 23 & +14.06 & 1 & +13.07 & +0.99 & +13.91 & +0.15 & 0.05 & +13.98 & +0.08 & 0.01 \\
\hline 24 & +18.89 & 1 & +18.56 & +0.33 & +18.96 & -0.07 & 0.22 & +19.12 & -0.23 & 0.01 \\
\hline 25 & $\begin{array}{r}+21.40 \\
\end{array}$ & 1 & $\begin{array}{r}+21.00 \\
\end{array}$ & +0.40 & $\begin{array}{r}+20.53 \\
\end{array}$ & +0.87 & 0.13 & +20.63 & +0.77 & 0.00 \\
\hline 26 & +20.37 & 1 & +20.86 & -0.49 & +20.20 & +0.17 & 0.08 & +20.27 & +0.10 & 0.00 \\
\hline 27 & +19.88 & 1 & +20.32 & -0.44 & +19.52 & +0.36 & 0.01 & +19.55 & +0.33 & 0.00 \\
\hline 28 & +15.17 & 1 & +16.23 & -1.06 & +15.48 & -0.31 & 0.14 & +15.40 & -0.23 & 0.00 \\
\hline 29 & -0.18 & 1 & -1.69 & +1.51 & -0.94 & +0.76 & 0.03 & -0.92 & +0.74 & 0.01 \\
\hline
\end{tabular}

Russell Tracy Crawford.

1901 July 24. 